\title{
Evaluation of the travel demand and proper solution suggestion for developing public bus transport: a case study in Ahwaz city
}

\author{
A. A. Davoodi ${ }^{1} \&$ S. A. Tabatabaie ${ }^{2}$ \\ ${ }^{1}$ Faculty of Engineering, Dezful Islamic Azad University, Iran \\ ${ }^{2}$ Faculty of Engineering, Shahid Chamran University, Iran
}

\begin{abstract}
This paper describes the evaluation of the travel demand and suggests a proper solution for developing public bus transport in Ahwaz city in Iran. Public bus transport is a multi-purpose public transportation system that is used widely for transporting passengers in medium and small cities. In the last decade, the development of cities and the increase in vehicles have caused environmental pollution and the traffic problems. Therefore, a sophisticated public transportation system should be designed. The problems with the present public bus transportation in Ahwaz city are discussed and bus travel demand is evaluated by using population data. In addition, the number of necessary public vehicles for solving passenger transport demand is estimated by using three methods.
\end{abstract}

Keywords: public transportation, travel, bus, urban transportation, demand.

\section{Introduction}

Traffic problems are increasing in large cities due to the increase in the amount of private cars. The development of roads and streets cannot be the proper solution to such problems [1]. Public transportation vehicles have several definitions and feature in various countries, such as countries that possess the owner vehicle culture (Japan, America, and Europe), and the others that do not [2]. The metro, LRT, tram, bus, and transportation systems have specific tasks. Buses as a basic ingredient of transportation systems in many world cities usually play a significant role in the traffic system. This system can be a suitable 
solution for a major group of citizens, if basic planning is designed, especially in cities whose population is under one million [3].

\section{The importance of public transportation}

The growth and development of cities, the weakness of public transportation systems, and the unfit planning of land use, make people use private cars. This phenomenon and the rush of drivers, particularly in central areas of the city, lead to most important problems in urban transportation. The number of private car owners was 184 per one thousand in 1970, and become 451 per thousand in 1997. This indicates a remarkable increase in private cars within a short duration [4].

Car congestion causes the following problems:

1) Increase in travel time when traveling by either private or public vehicles.

2) Pollution (air, noise).

3) Increase of risk of traffic security threat.

4) Lack of enough urban spaces for parking in parking places and on streets.

As presented above, using private cars instead of public vehicles results in increased air pollution. Studies carried out in Lisbon show:

1) Fuel consumption of private cars is six times more than that of public vehicles (buses).

2) The spread of CO and VOC gases by private cars is two to three times more than that of the buses.

3) Private vehicles produce more NOx and PM gases than buses [5].

Table 1 shows the spread of pollution in Ahwaz city based on the latest studies. It is obvious that the air pollution caused by the private cars is much more than the other transport vehicles. It can be concluded that a considerable portion of pollution caused by traffic in Ahwaz is because of private cars, especially in the center of the city. It can also be noticed that the pollution produced by the bus system, compared to others, is insignificant.

Table 1: $\quad$ Spread of pollution in Ahwaz (kg per day) [6].

\begin{tabular}{|c|c|l|c|c|c|}
\hline Vehicle & CO & HC & NOx & SOx & Particles \\
\hline Private car & 464877 & 1993 & 17931 & 8367 & 1593 \\
\hline Taxi & 23625 & 102 & 912 & 4253 & 87 \\
\hline Minibus & 260 & 605 & 972 & 792 & 476 \\
\hline Bus & 333 & 565 & 908 & 739 & 444 \\
\hline Motor cycle & 60550 & 259 & 2335 & 10899 & 225 \\
\hline
\end{tabular}

Therefore, it should be born in mind that the use of private cars has to be limited, and more attention should be paid to public transportation [7]. 


\section{Present condition of the bus system in Ahwaz}

Ahwaz is one of the seven largest cities in Iran. The population of Ahwaz is $1,100,000$ persons and it is located in the southwest of the country [8]. The pub bus transport network was established in 1970 with ten buses. Ahwaz has 79 bus lines in two zones whose length is $1659 \mathrm{~km}$. The network is so planned that lines of zone 1 compose the east section and lines of zone 2 compose the west section of the Karoon River. The total number of both public and private buses involved in the transportation system of the city is 199 [9]. The summary of general specifications of the bus system of Ahwaz is given in Table 2.

The Ahwaz bus system has three terminals. 28 of the 79 bus network lines end in one of the terminals (about 35\%) [9]. As in many other cities, the origin of most lines is located in the center of the city and the lines route comes out from the center in a radial shape. Statistics indicate that 224,000 persons are transported daily by buses.

Table 2: $\quad$ General specification of the bus lines in Ahwaz (2005) [9].

\begin{tabular}{|c|c|c|c|c|c|c|}
\hline \multirow{2}{*}{ Bus zones } & \multicolumn{3}{|c|}{ Number of lines } & \multicolumn{3}{c|}{ Line length (KM) } \\
\cline { 2 - 7 } & Public & Private & Total & Public & Private & Total \\
\hline 1 & 24 & 18 & 42 & 466 & 341 & 807 \\
\hline 2 & 21 & 16 & 37 & 367 & 485 & 852 \\
\hline Total & 45 & 34 & 79 & 833 & 827 & 1659 \\
\hline
\end{tabular}

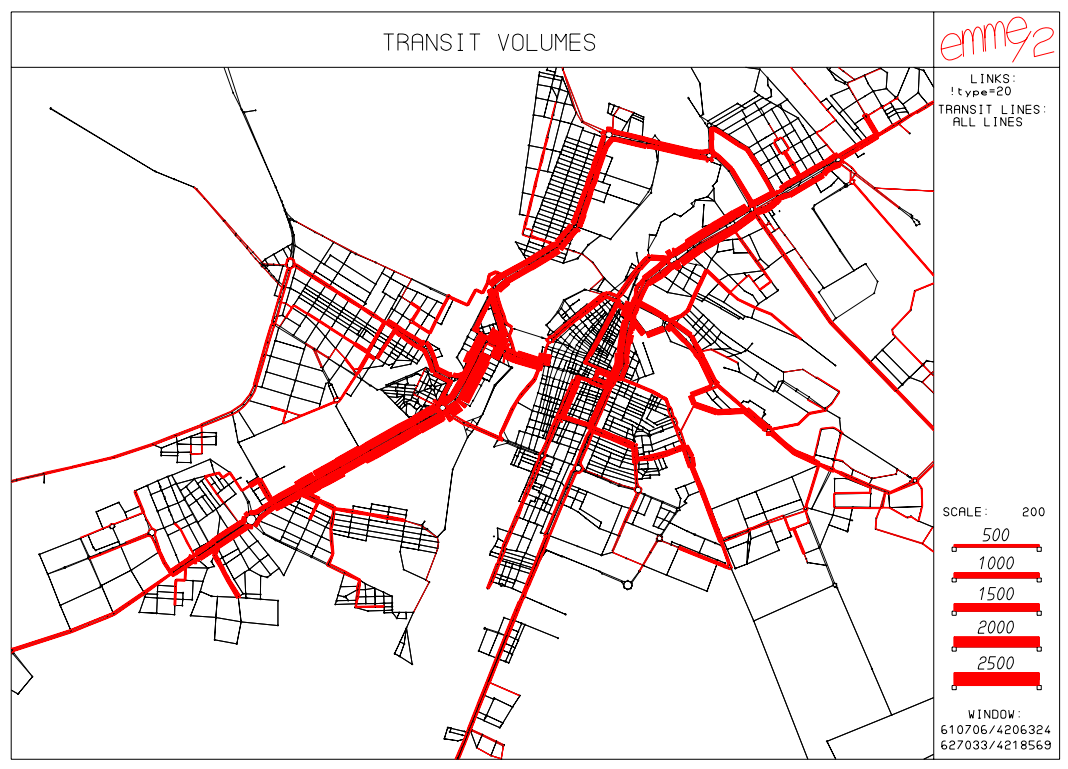

Figure 1: Assignment of the bus demand in the present conditions. 
The most and the least transported passengers in one route are 2,184 and 187 persons/day, respectively. The mean speed of the lines is $21.6 \mathrm{~km} / \mathrm{h}$, and the maximum and the minimum speeds are 45.6 and $11.5 \mathrm{~km} / \mathrm{h}$, respectively [9]. The world advanced standards suggest a mean speed of $36 \mathrm{~km} / \mathrm{h}$ [4]. The average time of movement of bus transportation is 11 hours/day, while it is 14 to 15 hours in other cities [9]. The reason for this difference is due to the extremely hot weather at midday (13-16) during the hot seasons in which the private lines do not operate. Figure 1 shows the assignment of bus demand in the present conditions.

\section{The bus transportation system problems and shortcomings}

In order to present a solution for the transportation system, firstly the problems and shortcomings should be identified. The problems are identified by interviews with people.

Interviews with people at stations and terminals indicate that the main problems are as follows:

1. The long waiting time at stations and terminals

2. The long stop of buses at final stations and terminals

3. Extremely hot weather for seven months of the year

4. Passengers rushing at peak hours

5. Unsuitable treatment by some bus drivers

6. Ignoring rules by some drivers

7. Some buses are our of order

8. Time schedules are ignored by some drivers

Many people are suffering because of a lack of buses and of long waiting times at stations. The above mentioned problems also result from the lack of expertise and management. The lack of the right management, absurd distribution of the lines, lapping of many lines together, multi-numbering of lines, severe lack of buses, absence of staff who run the system, for various reasons, absence of a suitable and planned structure in the daily work of this system, lack of educated and creative experts, absence of extensive use of new tools and technology, absence of sufficient defined rules for the private section, absence of the proper data recording for processing and managing data correctly and the use of traditional and nonsystematic methods in the management and guidance of this system altogether result in the above-mentioned problems and have led to the fact that the Ahwaz bus system is the worst compared to other cities in the country.

\section{Plans, bills and recognition of public transportation in country planning}

After recognition of the problems, identifying laws for the development and improvement of the public transportation is of major significance. According to the fourth development program (2005-2010) in the country, a share of the public transportation should reach $75 \%$ of the urban cities travellers. In addition, 
considering about 1,100 million dollars and 4 billion dollars finance for the provision of 6,500 buses according to national standards, providing repayment to buy Metro coaches, buying double fuel systems and empowering to devolution of a part of the bus lines to the private section are among the government actions in devolution finance to urban cities transportation [10]. However, the objectives of the fourth development program will not be achieved by the year 2010 .

\section{The travel demand evaluation of the public transportation system in five years}

Necessary arrangements and travel demands in present conditions are evaluated in this part of the study. Travel demands in the present situation are obtained from statistics that were compiled in December 2007.

\subsection{The travel demand evaluation by using method 1}

The Number of travels per day for buses in Ahwaz city according to statistics is 224,000 . The bus accounts for a share of about $14 \%$ of the whole daily travels in 2007 [9]. The population of Ahwaz in year 2005 was 1,188,000 persons and the travel rate is 1.36. Therefore, the total travels are estimated to be $1,615,000$ persons in this city [10]. If we suppose that the travel portion by different transportation methods and the mean travels number remain constant, for the estimated population at 2012 , which is $1,404,000$ persons, the total travels will be $1,909,440$ and the bus portion will be 267,000 travels.

\subsection{Travel demand evaluation by using method 2}

By using population data and travel rates, total travel demand in one 24 hour period can be obtained. So, the Ahwaz population in 2012 will be 1,404,000 persons, and by considering a travel rate index in 2012 at 1.6, total travel in one 24 hour period will be 2,246,000 persons [10]. By considering foregone government plans that allocate $15 \%$ of the urban cities travels to Metro and $35 \%$ of the total travels to buses and Minibuses, the travel numbers that will be made by Metro and bus are 337,000 and 786,000, respectively at plan year [10].

\section{Estimating the requisite buses}

The requisite buses can be calculated by the following parameters; the population, the journeys, and the defined measures by the cities supreme board secretariat.

\subsection{Estimating the requisite buses according to obtained demand by using method 1}

According to the above mentioned discussion, bus journeys in 2005 and 2012 are 224,000 and 267,000, respectively. Otherwise, each bus should transfer 900- 
1100 passengers a day [11]. Hence the number of active buses in 2005 (basic year) and 2010 (design year) are forecast to be 224 and 267 buses, respectively. If $15 \%$ of the buses are considered to be non-active, the number of requisite buses in the basic and design years will be 264 and 314 buses, respectively.

\subsection{Estimating requisite buses according to demand obtained by using method 2}

The public transportation portion of urban cities travels in cities with a population of more than one million persons during the fourth development program is shown in Table 3.

Table 3: Public transportation portion of the urban cities trips, in the beginning and the end of the fourth development program [12].

\begin{tabular}{|l|l|l|}
\hline Public Transportation System & 2005 & 2010 \\
\hline Metro & $1.8 \%$ & $15 \%$ \\
\hline Bus and Minibus & $28.7 \%$ & $35 \%$ \\
\hline Taxi and Private Cars & $22.3 \%$ & $25 \%$ \\
\hline
\end{tabular}

Considering linear growth of the portion of buses and minibuses in the period between 2005 and 2010, the portion of urban cities travels in 2005 and 2010 will be $31.2 \%$ and $35 \%$, respectively. According to Table 3, public transportation in 2005 is $28.7 \%$, the bus portion is $21.3 \%$ and the minibus portion is $5 \%$. Assuming the same trend until 2012, the bus portion at 2005 and 2012 will be $23.2 \%$ and $26 \%$, respectively. The minibus portion will be $8 \%$ and $9 \%$, respectively. Considering the Ahwaz population in 2005 and 2012, by using a daily rate of 1.36 trips per person in 2004, and 1.6 trips per person in 2012, the travel numbers in a 24 hour period will be $1,667,000$ and 224,600 , respectively and the bus portion will be 386,700 and 584,000 , respectively. The requisite bus number by considering $15 \%$ for non-active buses will be 455 and 687 buses, respectively.

\subsection{Estimating the requisite buses on the base of population $(\operatorname{method} 3)$}

Another way to evaluate requisite buses is by using the population parameter. Table 4 shows the bus numbers for every 100 thousand persons for the years from 1999 to 2004 .

Table 4: $\quad$ The bus number for every 100 thousand person from 1999 to 2004 [12].

\begin{tabular}{|l|l|l|l|l|l|l|}
\hline 2004 & 2003 & 2002 & 2001 & 2000 & 1999 & Year \\
\hline 36.4 & 35.1 & 33.6 & 32.5 & 31.3 & 29.7 & $\begin{array}{l}\text { The Required } \\
\text { Bus Number }\end{array}$ \\
\hline
\end{tabular}


Considering the bus number increases for 100 thousand persons, during the above mentioned years and assuming the growth to be linear, the bus numbers for every 100 thousand persons in 2006 and 2012 should be 39.1 and 43.1, respectively.

By using population measures, the active requisite bus numbers for 2006 and 2012 should be 479 and 605 , respectively and by considering $15 \%$ non-active buses, the requisite bus numbers will be 564 and 712, respectively.

\subsection{Comparison of results by the three methods}

To contrast the requisite bus number shows that the bus numbers evaluated by two methods, by using population and demand measures are very close to method 2. Thus, the real requisite bus number for Ahwaz city should be between these figures. Then, the requisite bus number considering the country development program, in year 2006, will be between 455 and 564, and in year 2012 should be between 687 and 712 buses, and evaluating the demand by method 1 yields for years 2006 and 2012, respectively 264 and 314 vehicles.

\section{Conclusion}

Determination of the optimal routes is the most major step in planning bus systems with optimal function. In designing route lines, proper spread network rules, minimum lapping of lines, absence of concentration of lines in one zone and proper line numbers should be considered. In order to improve the bus network the following alternatives are suggested:

Step 1 - It is proposed to eliminate long lines that end out of the city, and minibus lines should be replaced.

Step 2 - It is proposed to investigate eliminated lines that have capacities of less than 800 passengers a day. Among the determined indexes documents of the traffic supreme board secretariat for country cities, the daily mean passengers transferring by each bus is evaluated at between $900-1100$ persons. With more notice and by lowering ideals, a decrease of about $10 \%$ will bring the result to 800. This point leads to the deletion of about 16 lines.

Step 3 - lines below 800 passengers by bus should be inspected carefully to combine some of these lines together.

Step 4 - Eliminating some lines, keeping the other lines, and increasing the number of buses for other lines. This will result in the attraction of more passengers. Figure 2 shows the assignment of bus demand to the proposed form.

Other suggestions to improve the bus system in Ahwaz are given below:

1. Take advice from educated and young experts in management as well as experienced experts.

2. Create a database office to update information.

3. Plan to achieve goals of the fourth development program, and the next programs.

4. Change the method of privatization from persons to legal groups.

5. Increase utilities for the system and practice necessary changes in the system chart. 


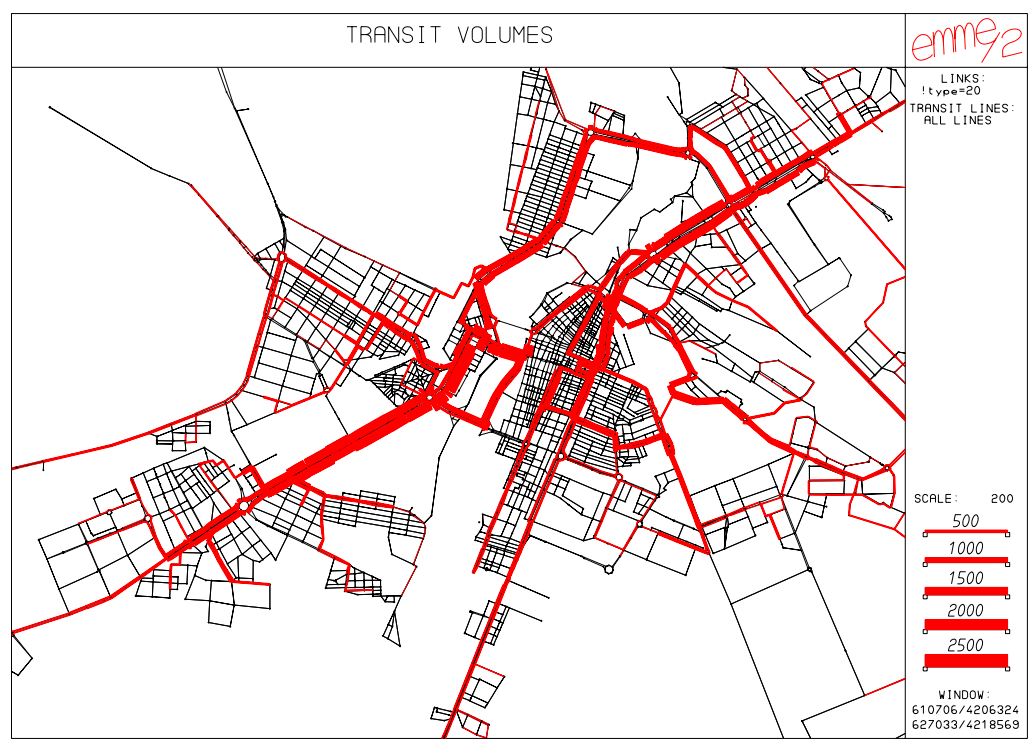

Figure 2: Assignment of bus demand to suggestible conditions.

\section{References}

[1] C. Taxiltaris, S. Basbas \& M. Miltiadou, Design and evaluation of traffic calming schemes in urban areas- the case of the city of Larissa, 2002

[2] Baha. A, Inspecting some of related technology with fitting civil express transportation, 2001

[3] Behbahani. H \& P. Hamed, Guidelines for urban street design, chapter 10, 2000 .

[4] Tabatabie. A, A. A. Davoodi, Public transportation a mean for decreasing traffic load in large cities, 2007

[5] Simoes. A. M, M. C. Coelho, C. M. Silva \& t. L. Farias, Analysis of the environmental impact of urban buses: application to a case study in Lisbon, 2002.

[6] Tabatabaie. S. A, Influencing factors on air pollution in Ahwaz, 2002.

[7] Jason. S. K, James. Y. L., C. K. Wang, The bus rapid transit system in Taipei city ridership forecast for the new light industrial area in Neihu, 2004.

[8] Lotfi. K, S. A. Tabatabaie, M. Tohidi, An analysis of Ahwaz bus services system and its place in urban transportation, as well as role it plays in reducing the traffic congestion within the central core of the city, 2004.

[9] Andishkar Consulting Company, Ahwaz traffic and transportation comprehensive studies, Report no 8, 2006.

[10] Andishkar Consulting Company, Ahwaz traffic and transportation comprehensive studies, Report no 19, 2006. 
[11] Organization of Country Mayoralty, Composition of evaluating civil bus system measures, 2005

[12] Transportation Office and Traffic Unity Supreme board Secretariat, "National document of urban civilization development", 2005 